\title{
Instantane Elemente und Oppositions-Ephemeride für (91) Aegina.
}

Die folgenden »instantanen* Elemente von (91) Aegina, welchen Planeten ich in meiner Theorie der kleinen Planeten als Rechnungsbeispiel gewählt habe, sind dem vierten Teil dieser Arbeit entnommen, der demnächst erscheinen wird. Über die Definition dieser Elemente, welche den oskulierenden hinsichtlich der Kürze der Rechnung erheblich überlegen sind, werde ich noch näheres mitteilen. Einstweilen bemerke ich nur, daß bei Berücksichtigung einiger Hauptstörungsglieder für Jupiter, welche ich für alle Planeten, deren mittlere Bewegung größer als 709" ist, tabuliert habe, die zur Vergleichung herangezogenen Beobachtungen der Aegina aus den Jahren 1866 bis 1907 so dargestellt werden, daß die größte Differenz in Rektaszension $2^{\mathbf{5}} \cdot 3$ und in Deklination $\mathbf{2 3}^{\text {" }}$ beträgt. Diese Übereinstimmung läßt nichts $\mathrm{zu}$ wünschen übrig, da sie sich innerhalb der (ienauigkeitsgrenze hält, nit der die Rechnung überhaupt ausgeführt worden ist.

Die Elemente sind (mittl. Äqu. I 900.0):

$\begin{array}{rc}1910 & \text { März 5.0 } \\ \log e & 9.02294 \\ \pi & 81.939 \\ L & 185.542 \\ \log p & 0.40831 \\ \log \sin i & 8.5710 \\ \delta & 10^{\circ} .92\end{array}$

April 14.0

9.02293

$81: 934$

195.013

0.40832

8.5710

10.92

Mai 24.0

9.02292

81.929

204.479

0.40834

8.5710

$10^{\circ} .92$

Mittlere Bewegung $=852$ ". 6

Mittlere Beschleunigung $=-0.0055$

Mittl. Länge $=195^{\circ}$.01 $3+85^{\prime \prime}$ 16 $6 t-0.0055 t^{2}$ $t$ gezählt in Tagen von 19 I 0 April $140^{\text {h }}$ M. Z. Berlin.

Obwohl der Ort des Planeten durch die Tafeln auf lange Zeit gesichert sein wird - meine Tafeln gehen bis zum Jahre r 950 - so mag doch die Oppositionsephemeride hier mitgeteilt werden, da es immer von Wert sein wird, eventuell noch Beobachtungen aus dieser Opposition zu erhalten. I $2^{\text {h }}$ M. Z. Berlin.

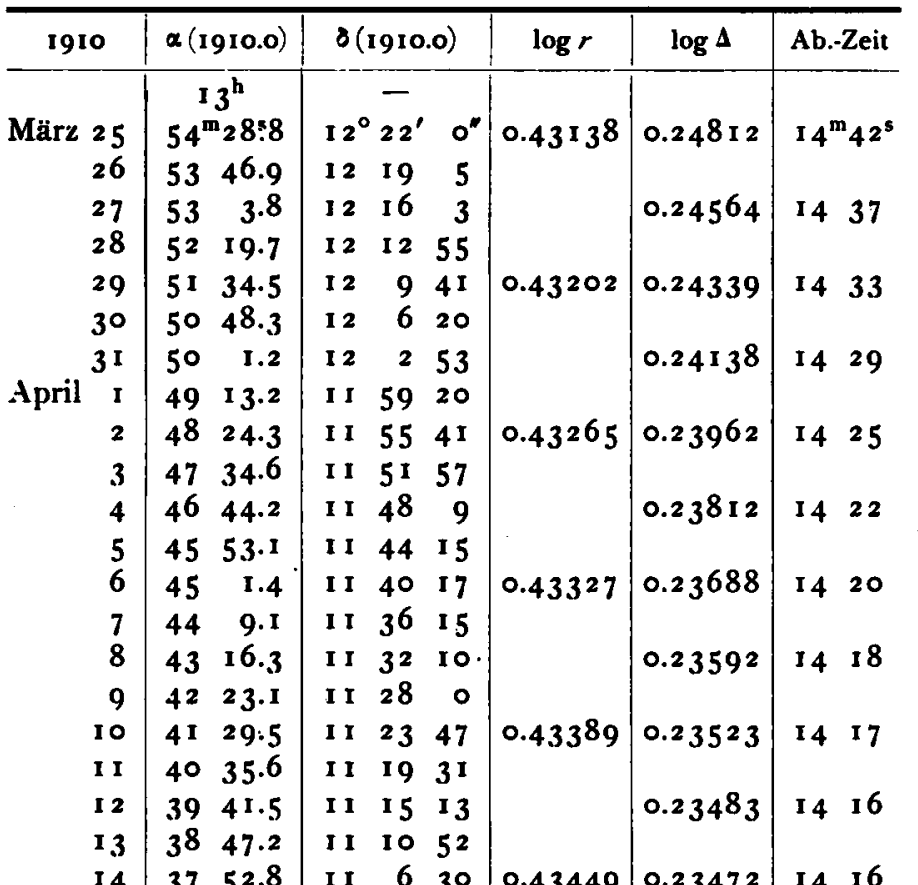

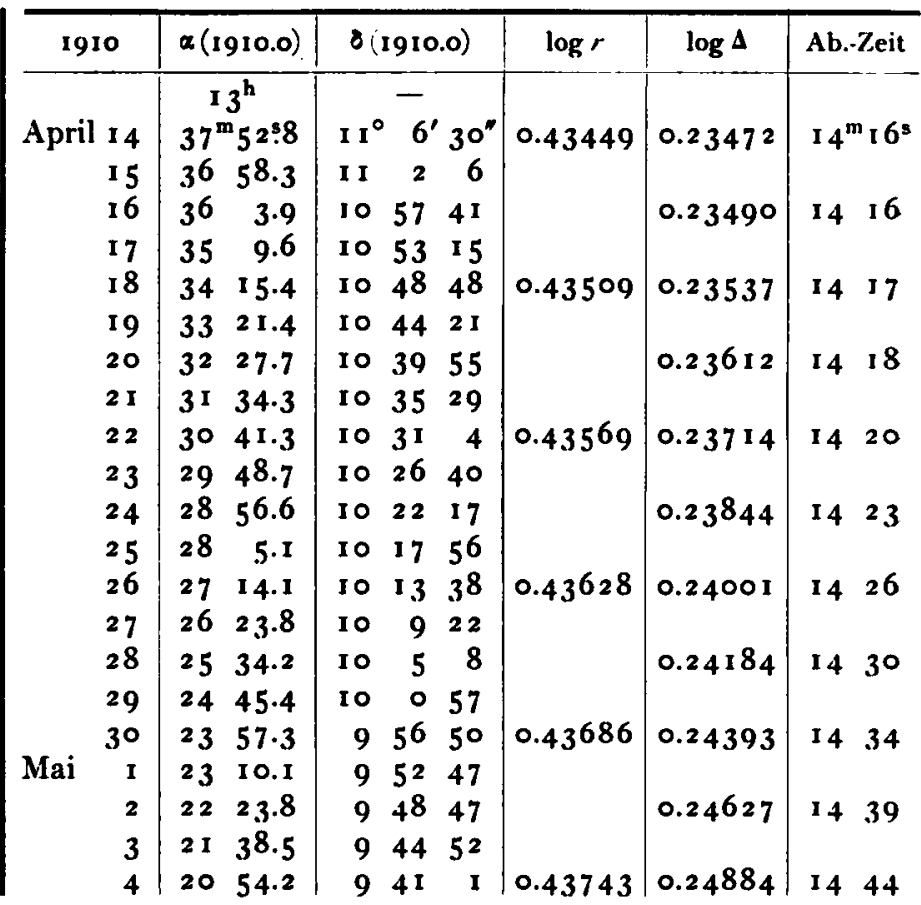

Opposition in RA: April 16. Größe $=11 \mathrm{mo}$.

Frankfurt a. M., I 9 I o März 4.

M. Brendel.

Komet 1909 e (Daniel). Der Komet wurde r 9 r o März 6 bei sehr reiner Luft im 27 -Zöller nicht mehr gesehen. F. Palisa.

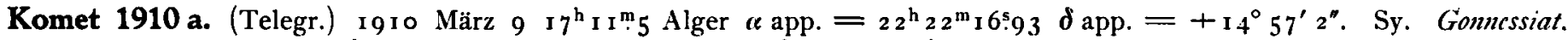
л9 го März $1216^{\mathrm{h}} 5^{\mathrm{m}} .8 \mathrm{Mt}$. Hamilton $c$ app. $=22^{\mathrm{h}} 24^{\mathrm{m}} 43^{\mathrm{s}} \mathrm{I} \delta$ app. $=+15^{\circ} 3^{\prime} 44^{\prime \prime}$. Aitken. Campbell.

Planet $1910 \mathrm{JW}$. März $78^{\mathrm{h}} 46^{\mathrm{m}} 20^{\mathrm{s}}$ Wien ce app. $=3^{\mathrm{h}} 3^{6^{\mathrm{m}}} 3^{\mathrm{s}} \cdot 35$ (9.575) $\delta$ app. $=+22^{\circ} 40^{\prime} 14^{\mathrm{h}} \cdot 7$ (o.697) Gr. I $2^{\mathrm{m}} \cdot \mathrm{o}$

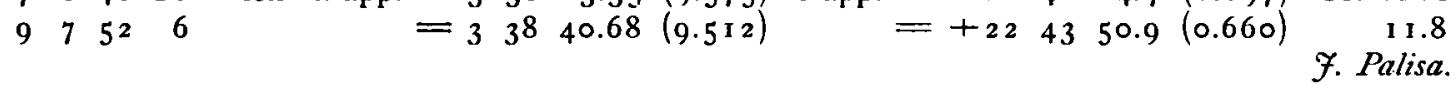

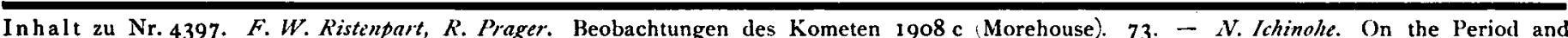
Light-Curve of a New Variable Star 26.1910 Scuti. 83. - M. Wolf. Photographische Aufnahinen auf der KönigstuhlSternwarte. 85. - Korrektionen von Planeten-Ephemeriden. 85. - .1. Brendel. Instantane Elemente und Oppositions. Ephemeride für (91) Aegina. 87. - Kleine Mitteilungen. 87. 